\title{
VARIATION OF DOMAIN FUNCTIONALS ${ }^{1}$
}

\section{SCHIFFER}

1. Introduction. Let $D$ be a domain in an $m$-dimensional space and $\phi[D]$ be a number which depends on the domain. We shall call $\phi[D]$ a domain functional. Insofar as $D$ is also characterized by its boundary $C$ or its complement $\widetilde{D}$, we may sometimes consider the functional $\phi[D]$ as dependent on $C$ or $\widetilde{D}$ instead of $D$. The problem with which we are occupied is to establish a calculus for the functional with respect to changes of its variable; in other words, a calculus of variations for $\phi[D]$.

We give the following examples of domain functionals: The volume $V[D]$ of the domain and the surface area $A[C]$ of its boundary which are elementary geometric concepts. Let $\nabla^{2}$ be the Laplace operator and consider the eigenvalue problem $\nabla^{2} u+\lambda u=0$ with the boundary condition $u=0$ on $C$. The eigenvalues $\lambda_{\nu}[D]$ of this problem are often studied domain functionals. The corresponding eigenfunctions $u_{\nu}(x)$ taken at a fixed point $x$ may be considered likewise as domain functionals. Finally, we mention the Green's function of $D$ with respect to Laplace's equation $G(x, \xi)$. If we consider both its argument points $x$ and $\xi$ as fixed parameters, it becomes a functional of $D$.

The first domain functionals of nonelementary character to be studied seem to have been the eigenvalues $\lambda_{\nu}(D)$ of a plane domain [17] and the electrostatic capacity of a three-dimensional conductor [15]. Since both these quantities are closely related to the Green's function of the domains considered with respect to Laplace's equation, their theory can be reduced to that of the Green's function. Hadamard treated $G(x, \xi)$ from the general point of view of functional analysis and gave for the first time a systematic basis to the whole problem complex [7].

In order to study the dependence of a functional on its variable, we have, in the spirit of classical analysis, to investigate how the functional changes under an infinitesimal change of the domain. Hadamard assumed that the domain $D$ is bounded by a closed smooth surface $C$. He deforms $C$ into a surface $C^{*}$ by pushing each point $\zeta$ on $C$ by an amount $\delta n$ in the direction of the exterior normal. Under

An address delivered before the Pasadena meeting of the Society on November 28, 1953, by invitation of the Committee to Select Hour Speakers for Far Western Sectional Meetings; received by the editors December 15, 1953.

1 This work was done under a contract with the Office of Naval Research. 
certain restrictive assumptions on $\delta n$, we can assert that $C^{*}$ is also a smooth surface which bounds a domain $D^{*}$ with a Green's function $G^{*}(x, \xi)$. If $\delta n>0, G^{*}(x, \xi)$ is well-defined in $D$ and, by Green's identity, we find

$$
\begin{aligned}
-\iint_{C}\left[G^{*}(\zeta, x) \frac{\partial G(\zeta, \xi)}{\partial n}-G(\zeta, \xi) \frac{\partial G^{*}(\zeta, x)}{\partial n}\right] d \sigma & \\
& =G^{*}(x, \xi)-G(x, \xi) .
\end{aligned}
$$

Now, $G(\zeta, \xi)=0$ on $C$ and

$$
G^{*}(\zeta+\delta \zeta, \xi)=G^{*}(\zeta, \xi)+\left(\partial G^{*} / \partial n\right) \delta n+\cdots=0 .
$$

Hence obviously

$$
\delta G(x, \xi)=\iint_{c} \frac{\partial G(\zeta, x)}{\partial n} \frac{\partial G(\zeta, \xi)}{\partial n} \delta n d \sigma .
$$

This is Hadamard's variational formula for the Green's function.

Though the above derivation is purely formal, it can be justified if the surface $C$ is regular enough and many interesting applications can be made. Since $\partial G / \partial n<0$ on $C$, we recognize, for example, that $\delta G>0$ if $\delta n>0$, that is the monotony of the Green's function in dependence of the domain. Our formula would, at first, guarantee this monotonicity only for domains with sufficiently regular boundary. But it is easy to extend this result to the most general domains, since every domain can be approximated arbitrarily by domains whose boundary is even analytic.

Much deeper results can be derived by similar considerations; each time when we have a functional of $D$ which is a combination of Green's functions and various partial derivatives of Green's functions such that the coefficient of $\delta n d \sigma$ in the variational formula is positive, we arrive at a monotonicity theorem for this functional.

Similar variational formulas can be easily established for the Green's functions of other partial differential equations of elliptic type, for higher orders, and for different boundary conditions. Hadamard studied, in particular, the Green's functions for the biharmonic equations which play a central role in the theory of elasticity of plates. But while the theory of boundary value problems in partial differential equations proceeded to more general domains, $D$, the variational method was inherently tied to a very restricted class of regular domains. Even very simple extremum problems for domain functionals could not be solved by variational methods, since one is not sure, a priori, that the sought extremum domain is regular 
enough to admit a variational formula. It is clear that the difficulty lies in the kinematics of our variation. Since we describe the transition from one domain to its neighbor by a normal shift, it is obvious that the normal at the varied points of $C$ will play a role in the variational formula, and that we shall, a priori, exclude all domains $D$ from the theory whose boundary $C$ does not possess a normal at each point.

2. Theory of univalent functions. The theory of domain functionals was applied in a precise and successful manner at first in the theory of conformal mapping. Let $\Delta$ be a domain in the complex z-plane which contains the point at infinity. We call a domain $D$ in the complex w-plane conformally equivalent to $\Delta$ if there exists in $\Delta$ a univalent analytic function which has near $z=\infty$ the development

$$
w=f(z)=z+a_{0}+\frac{a_{1}}{z}+\cdots
$$

and maps $\Delta$ onto $D$. Since $D$ determines $f(z)$ in a unique way, we may consider all coefficients $a_{\nu}[D]$ as domain functionals of $D$ and pose various extremum problems with respect to them. de Possel [16] asked for a domain $D$ which is conformally equivalent to $\Delta$ and for which

$$
\operatorname{Re}\left\{a_{1}[D]\right\}=\max .
$$

The existence of such a domain is insured by theorems on the normality of the family of univalent functions with the development (2.1) at infinity. There is only the question of characterizing the extremum function. de Possel showed that the extremum domain $D$ consists of the whole w-plane slit along rectilinear segments parallel to the real axis. Since the existence of the extremum domain is sure, this constitutes an existence proof for a canonical conformal mapping. Since de Possel's proof many other canonical mappings have been derived in the same way. The preceding reasoning shows the great value of the variational method for the existence proof in complicated boundary value problems. The method is to be clearly distinguished from extremum methods as, for example, Dirichlet's principle. There we work with a fixed domain and a very wide class of admissible functions, while in our case we are dealing only with analytic univalent functions but varying domains.

Another classical problem in conformal mapping deals with the family of all functions

$$
f(z)=z+a_{2} z^{2}+\cdots+a_{n} z^{n}+\cdots
$$


which are univalent in the unit circle $|z|<1$. This family plays an important role in the general theory of conformal mapping and uniformization. It is a normal family which implies again that each sensible extremum problem has a solution, that is, at least one extremum function. The classical problem of the theory, as yet unsolved, is the question of $\max \left|a_{\nu}\right|$ for the class $\mathfrak{F}$. Bieberbach's conjecture

$$
\left|a_{\nu}\right| \leqq \nu
$$

which is proved for $\nu=2,3$ attracted the attention of many mathematicians because of its great simplicity.

Each function $f(z) \subset F$ determines a domain $D$ in the complex plane which may be considered as the graphical representation of $f(z)$ and, conversely, $f(z)$ and its coefficients $a_{\nu}$ may be conceived as functionals of $D$. Let now $\phi(z)$ be the inverse function of $f(z)$ which maps $D$ onto the unit circle; then

$$
-\frac{1}{2 \pi} \log |\phi(z)|=G(z, 0)
$$

is easily seen to be the harmonic Green's function of $D$ with the source point at the origin. Thus, the coefficient problem for univalent functions may be considered as an extremum problem for the Green's function of a domain $D$. That this point of view is natural is seen from the following fact. The function $\phi(z)$ which is nearer to the Green's function than its inverse $f(z)$ also gives rise to a coefficient problem. This problem is simpler than the preceding one and was solved completely by Löwner in 1923 [11]. Thus, the problem closer to the Green's function problem seems for this reason to be easier to handle.

In his classical paper of 1923 Löwner considered a sequence of varying domains which depend on a parameter $t$ as follows. Let $\Gamma$ be a Jordan arc $z(t)$ which runs from $z(0)$ to infinity as $t$ runs from zero to infinity. Let $\Gamma_{t}$ be the subarc of $\Gamma$ between $z(t)$ and infinity and let

$$
f(z, t)=\sum_{\nu=1}^{\infty} a_{\nu}(t) z^{\nu}
$$

be the function which maps the unit circle onto the complex plane slit along the $\operatorname{arc} \Gamma_{t}$. Then, $f(z, t)$ is differentiable in $t$ and satisfies a simple and elegant first order partial differential equation in $z$ and $t$. On the other hand, Julia [9] transformed Hadamard's variational formula for the Green's function into a variational formula for the corresponding mapping function $f(z)$ and Biernacki [1] showed that 
Löwner's differential equation follows from Julia's formula by a proper passage to the limit. Thus, one of the most successful methods in the theory of univalent functions was tied to Hadamard's variational method.

In the special case of the Laplace equation in two variables there exists a simple device in order to extend Hadamard's formula to general domains and thus to make it applicable to extremum problems. This device is based on the fact that a harmonic function in two variables remains harmonic under conformal mapping; if $u(z)$ is harmonic in $x, y(z=x+i y)$, then $u[f(\zeta)]$ is harmonic in $\xi, \eta(\xi=\xi+i \eta)$. Let now $D$ be a domain in the $z$-plane with finitely many analytic boundary curves $C$; let $\Delta$ be a subdomain interior to $D$ with finitely many analytic boundary curves $\Gamma$. Let $f(z)$ be regular analytic outside of $\Delta$; then $z^{*}=z+\epsilon f(z)$ will be regular analytic on $C$ and, for small enough $\epsilon$, even univalent. Hence, the curve system $C$ will be mapped onto a new curve system $C^{*}$ which determines a new domain $D^{*}$ with the Green's function $G^{*}(z ; \zeta)$. Clearly

$$
g_{\epsilon}(z, \zeta)=G^{*}[z+\epsilon f(z), \zeta+\epsilon f(\zeta)]
$$

is harmonic in $D-\Delta$ for $z \neq \zeta$ and vanishes for $z$ or $\zeta$ on $C$. We assume that $z, \zeta$ lie in $D-\Delta$ and apply Green's identity:

$$
g_{\epsilon}(z, \zeta)-G(z, \zeta)
$$

$$
=\int_{C+\Gamma}\left[g_{\epsilon}(t, \zeta) \frac{\partial G(t, z)}{\partial n}-G(t, z) \frac{\partial g_{\epsilon}(t, \zeta)}{\partial n}\right] d s .
$$

Since $G(t, z)$ as well as $g(t, \zeta)$ vanishes on $C$, there remains only the integration over the curve $\Gamma$, interior to $D$.

Formula (2.8) was derived for a domain $D$ with analytic boundary $C$; but now we may extend this formula to the most general case $C$ by approximating $C$ by analytic curves. Using theorems on the uniform convergence of the corresponding Green's functions in a closed subdomain of $D$, we can justify the above result for the general case.

The law of deformation

$$
z^{*}=z+\epsilon f(z)
$$

defines to the most general boundary $C$ of a domain $D$ a new boundary $C^{*}$ if $C$ lies outside of $\Delta$ and if $\epsilon$ is small enough. For most applications it is sufficient to choose $f(z)$ in a very special way, namely,

$$
f(z)=\frac{1}{z-z_{0}}, \quad z_{0} \in D .
$$


Using variations of this type, numerous extremum problems in the theory of univalent functions could be treated $[6 ; 19 ; 20 ; 21]$. One of the simplest and most important results states that the extremum functions of the coefficient problem are analytic on the periphery of the unit circle with the exception of finitely many points.

More precisely, a function $f(z)$ belonging to a maximal value of $\left|a_{n}\right|$ satisfies an ordinary differential equation

$$
f^{\prime}(z)^{2} P_{n}\left[f(z)^{-1}\right]=z^{-(n+1)} Q_{n}(z)
$$

where $P_{n}$ and $Q_{n}$ are polynomials whose coefficients depend in a specified manner on the first $n$ coefficients of $f(z)$ itself. It can be shown that the function

$$
f(z)=\frac{z}{(1-z)^{2}}=z+2 z^{2}+\cdots+n z^{n}+\cdots,
$$

which is the conjectured extremum function for all values of $n$, satisfies the above functional-differential equation.

Teichmüller has proved a converse of the above theorem [23]. Namely, suppose that a univalent function $f(z)$ satisfies a differential equation of the type (2.11) with any polynomial

$$
P_{n}(x)=\alpha_{1} x+\alpha_{2} x^{2}+\cdots+\alpha_{n-1} x^{n-1}, \alpha_{n-1}=1 ;
$$

then $\operatorname{Re}\left\{a_{n}\right\}$ will be maximal for this function with respect to all functions of the family $\mathfrak{F}$ which have the same first $n-1$ coefficients as $f(z)$. This result shows that every univalent solution of (2.11) is in some sense an extremum function. However, in order to decide whether $f(z)$ is the extremum function of some specific extremum problem, more powerful methods seem to be needed.

It is natural to expect additional information from a theory of the second variation for the functionals considered. Since the vanishing of the first variation ensures already the analyticity of the boundary of the extremum domain, it seems sufficient to establish a formula for the second variation in the Hadamard kinematics. However, the computational difficulties in deriving the second variation for the Green's function are considerable; the formula was established only very recently and will be given in $\$ 4$.

3. General interior variations. The variational kinematics used in the preceding section can be easily applied to much more general domain functionals. In particular, it permits us to deal with functionals which are connected with boundary value problems for selfadjoint elliptic differential equations. For the sake of simplicity, we 
shall illustrate the method by treating Laplace's equation in threespace.

We define in the space with coordinates $x_{i}(i=1,2,3)$ a vector field $S_{i}(x)$ which is twice continuously differentiable and consider the transformation of the whole space

$$
x_{i}^{*}=x_{i}+\epsilon S_{i}(x)
$$

which depends on the real parameter $\epsilon$. If $\epsilon$ is small enough, this transformation will be univalent in a given sphere of radius $R$. Every domain $D$ in this sphere is mapped topologically onto a domain $D^{*}$ and we denote the corresponding harmonic Green's functions by $G(x, \xi)$ and $G^{*}(x, \xi)$, respectively. Our problem is to express $G^{*}(x, \xi)$ in terms of $G(x, \xi)$ and of the transformation vector field. We shall call the deformation of a domain by means of such a vector field an interior variation.

In order to study $G^{*}(x, \xi)$, we consider the function

$$
g(x, \xi ; \epsilon)=G^{*}\left(x^{*}(x), \xi^{*}(\xi)\right)
$$

which is defined in the original domain $D$, is twice continuously differentiable there if $x \neq \xi$, and which vanishes if $x$ or $\xi$ lies on the boundary $C$ of $D$. In the case of harmonic functions in the plane, we utilized a deformation vector field $S_{i}(x)$ of particular type, namely, we chose $S_{i}$ so that $f(z)=S_{1}+i S_{2}$ was an analytic function of $z=x_{1}$ $+i x_{2}$ outside of some subregion $\Delta$. This particular choice had the advantage that $g(x, \xi ; \epsilon)$ was still harmonic in $D$ outside of $\Delta$ for arbitrary choice of $\epsilon$. Now, the situation is more difficult; we cannot preserve the harmonicity of $G(x, \xi)$ under the deformation, but we are led to a new differential equation for $g(x, \xi ; \epsilon)$ which expresses that $G^{*}\left(x^{*}, \xi^{*}\right)$ is harmonic in $D^{*}$. In fact, a simple calculation shows that Laplace's equation in $D^{*}$ is translated into

$$
\sum_{i=1}^{3} \frac{\partial}{\partial x_{i}}\left(\sum_{k=1}^{3} A_{i k} \frac{\partial}{\partial x_{k}} g(x, \xi ; \epsilon)\right)=0
$$

with

$$
A_{i k}=A_{k i}=\theta \sum_{j=1}^{3} \frac{\partial x_{i}}{\partial x_{i}^{*}} \frac{\partial x_{k}}{\partial x_{j}^{*}}, \quad \theta=\frac{\partial\left(x_{1}^{*}, x_{2}{ }^{*}, x_{3}{ }^{*}\right)}{\partial\left(x_{1}, x_{2}, x_{3}\right)}
$$

We observe that the coefficients of the new differential equation depend analytically on $\epsilon$.

It is clear that $g(x, \xi ; \epsilon)$ and $G^{*}(x, \xi)$ are equivalent functions and that the knowledge of one leads to the other in an elementary way. 
We have expressed the Green's function $G^{*}(x, \xi)$ for the fixed Laplace equation and a variable domain $D^{*}$ in terms of the Green's function $g(x, \xi ; \epsilon)$ for a varying differential equation (3.3) but with respect to the original fixed domain $D$. This was achieved by transplanting $G^{*}(x, \xi)$ back into $D$ by use of the inverse transformation of (3.1).

The dependence of the solution of a partial differential equation $L_{\epsilon}[\imath]=0$ with fixed boundary value on the parameter $\epsilon$ occurring in the coefficients of the equation has been frequently investigated. Hilbert applied the parametrix method successfully for this problem [8]. It can be shown [3] that in our particular case the Green's function $g(x, \xi ; \epsilon)$ depends analytically on the parameter $\boldsymbol{\epsilon}$.

We may develop $g$ into a power series in $\epsilon$ and calculate without difficulty the coefficients of this development. These are clearly closely related to the variations of the respective orders of the Green's function $G(x, \xi)$. We find, for example:

$$
\left.\frac{\partial}{\partial \epsilon} g(x, \xi ; \epsilon)\right|_{\epsilon=0}=\iiint_{D} \sum_{i, k=1}^{3} T_{i k}(\eta ; x, \xi) \frac{\partial S_{i}(\eta)}{\partial \eta_{k}} d \eta_{1} d \eta_{2} d \eta_{3}
$$

with

$$
\begin{aligned}
T_{i k}(\eta ; x, \xi)= & \frac{\partial G(\eta, x)}{\partial \eta_{i}} \frac{\partial G(\eta, \xi)}{\partial \eta_{k}}+\frac{\partial G(\eta, x)}{\partial \eta_{k}} \frac{\partial G(\eta, \xi)}{\partial \eta_{i}} \\
& -\delta_{i k}\left(\nabla_{\eta} G(\eta, x) \cdot \nabla_{\eta} G(\eta, \xi)\right) .
\end{aligned}
$$

The tensor $T_{i k}(\eta ; x, \xi)$ plays a central role in the variational theory of the Green's function. It may be called the Maxwell tensor of $D$; if we put $x=\xi$, we see in fact that $T_{i k}(\eta ; x, x)$ is the classical Maxwell tensor of the electrostatic field created in the domain $D$ with grounded conducting walls $C$ by a unit charge at the point $x \in D$.

The tensor $T_{i k}$ is obviously symmetric in its indices as well as in $x$ and $\xi$. It satisfies the divergence condition

$$
\sum_{k=1}^{3} \frac{\partial}{\partial \eta_{k}} T_{i k}(\eta ; x, \xi)=0, \quad i=1,2,3 .
$$

This identity indicates that formula (3.4) can be simplified considerably by use of the divergence theorem; we can reduce the triple integral into an integral over the surface $C$ of $D$ provided that $C$ is regular enough to admit the application of the divergence theorem. We have to observe in the transformation of the integral that $T_{i k}$ becomes infinite for $\eta=x$ and $\eta=\xi$ and have to consider the residues from these singular points. Carrying out all the indicated steps, we arrive at 


$$
\left.\frac{\partial}{\partial \epsilon} G^{*}(x, \xi)\right|_{\epsilon=0}=\iint_{C} \frac{\partial G(\eta, x)}{\partial n} \frac{\partial G(\eta, \xi)}{\partial n}(S \cdot n) d \sigma
$$

which is exactly Hadamard's variational formula (1.2). We obtain in this way a new and precise proof for Hadamard's classical formula under the weakest assumptions. On the other hand, (3.4) appears now clearly as the generalization of this formula to the case of arbitrarily bounded domains $D$.

A completely analogous formula holds also in the case of twodimensional harmonic Green's functions. In this case it happens that the trace $\sum_{i} T_{i i}$ of the Maxwell tensor vanishes identically. Hence, $T_{i k}$ has only two essential components, and we have

$$
T_{11}=-T_{22}, \quad T_{12}=T_{21} .
$$

If we choose now the vector field $S_{i}(x)$ in such a way that

$$
\frac{\partial S_{1}}{\partial x_{1}}=\frac{\partial S_{2}}{\partial x_{2}}, \quad \frac{\partial S_{1}}{\partial x_{2}}=-\frac{\partial S_{2}}{\partial x_{1}}
$$

we see that the integrand in (3.4) vanishes identically. This means, of course, the invariance of the Green's function under conformal mapping. It is now clear why the method of interior variation is particularly successful in the two-dimensional case; by choosing $S$ to be an analytic vector field in the whole plane except for isolated singular points, we can express the variation of the Green's function in terms of its values and the values of its derivatives at these distinguished critical points. Extremum conditions on the Green's function which have, in general, the form of integro-differential equations reduce in this case to ordinary differential equations.

We illustrate the general variational method in a relatively simple case. We consider a plane, finite, and simply-connected domain $D$ with boundary $C$ and the eigenvalue problem

$$
\nabla^{2} u+\lambda u=0, \quad u=0 \text { on } C .
$$

Let $z_{0}$ be the complex coordinate of a point in the $z$-plane but not on $C$. We use the deformation

$$
z^{*}=z+\frac{\epsilon}{z-z_{0}},
$$

with complex parameter $\epsilon$, which is univalent in $\left|z-z_{0}\right|>|\epsilon|^{1 / 2}$ and which maps therefore, for small enough $\epsilon, D$ into a domain $D^{*}$. From the general variational procedure, we find that each eigenvalue $\lambda(D)$ 
which is nondegenerate corresponds to an eigenvalue $\lambda\left(D^{*}\right)$ by the formula [4]

$$
\begin{aligned}
\lambda\left(D^{*}\right)=\lambda(D)-\operatorname{Re} & \left\{8 \pi \epsilon \delta\left(z_{0}\right)\left(\frac{\partial u\left(z_{0}\right)}{\partial z_{0}}\right)^{2}\right. \\
& \left.-2 \lambda(D) \epsilon \iint_{D} \frac{u(z)^{2}}{\left(z-z_{0}\right)^{2}} o \omega\right\}+o(\epsilon) .
\end{aligned}
$$

Here $\delta\left(z_{0}\right)=0$ or 1 if $z_{0}$ lies in the exterior or interior of $D$, respectively, and

$$
\frac{\partial}{\partial z_{0}}=\frac{1}{2}\left(\frac{\partial}{\partial x_{0}}-i \frac{\partial}{\partial y_{0}}\right) .
$$

$o(\epsilon)$ can be estimated uniformly for all domains $D$ whose boundaries stay outside of a fixed circle $\left|z-z_{0}\right|=\alpha$. The above formula applies, in particular, always to the first eigenvalue $\lambda_{1}(D)$ which is known to be nondegenerate.

Under the same variation (3.10) the area $A(D)$ is transformed into

$$
A\left(D^{*}\right)=A(D)-\operatorname{Re}\left\{2 \epsilon \iint \frac{d \omega}{\left(z-z_{0}\right)^{2}}\right\}+o(\epsilon) .
$$

We may now treat Rayleigh's problem to find the minimum for the product $A \cdot \lambda_{1}$. The existence of a minimum domain can be derived by use of the conformal mapping function which carries $D$ into the unit circle and applying the normality of the family of univalent functions. We can characterize the extremum domain by the fact that the first variation of $\lambda_{1} A$ has to vanish for each choice of $\epsilon$ and $z_{0} \notin C$. This leads to the functional equation

$$
\delta\left(z_{0}\right)\left(\frac{\partial u\left(z_{0}\right)}{\partial z_{0}}\right)^{2}=\frac{\lambda_{1}(D)}{4 \pi A(D)} \iint_{D} \frac{A(D) u(z)^{2}-1}{\left(z-z_{0}\right)^{2}} d \omega .
$$

By a careful analysis of this condition one can show that $D$ has an analytic boundary curve $C$ along which $\partial u / \partial n=$ const. We obtain by the variational method less than from the more elementary method of symmetrization which has been developed in great elegance by Pólya-Szegö [13]. However, the same variational technique can be applied in much more general problems where the extremum domain will not happen to be symmetric and the method will work as well.

In $\$ 2$, we pointed out that the variational theory of domain functionals leads often to important existence theorems. We want to discuss here a problem which plays a role in applications. Let $B$ be a 
body in three-space, considered immersed in an incompressible nonviscous fluid of density 1 . Suppose that the fluid stream past the body is a stationary irrotational flow with velocity one at infinity and direction parallel to the $x$-axis. We denote the velocity potential of the flow by $\phi(x, y, z)$; it is a harmonic function in the domain $D$ outside of $B$ except at infinity and is at each fixed point in $D$ a domain functional of $B$ (or $D$ ). If we split off from $\phi(x, y, z)$ the singular term due to the source of the flow at infinity, we can write it in the form

$$
\phi(x, y, z)=-x+\varphi(x, y, z)
$$

where $\varphi$ is regular harmonic in $D$. The quantity

$$
M=\iiint_{D}(\nabla \varphi)^{2} d x d y d z
$$

is called the virtual mass of $B$ and plays a central role in the hydromechanics of $B ; M$ is also a functional of $B$.

Suppose now that we deform the surface $C$ of $B$ into a surface $C^{*}$ of $B^{*}$ by a normal shift $\delta n$ applied to all its points. It can be shown [22] that

$$
\delta M=\iint_{C}(\nabla \phi)^{2} \delta n d \sigma-\delta V,
$$

where at the same time the volume $V(B)$ varies according to

$$
\delta V=\iint_{C} \delta n d \sigma .
$$

We apply these results to an important problem in fluid dynamics, namely, the free boundary problem. Helmholtz was the first to point out that the flow past a body $B$ is by no means uniquely determined. The moving fluid may bypass the body $B$ and leave also a part of the fluid itself adjacent to $B$ at rest. The part of the fluid at rest together with the body $B$ forms a larger body $B^{\prime}$ whose velocity potential $\phi^{\prime}(x, y, z)$ determines the correct velocity field of the flow. However, since the hydrostatic pressure in the resting fluid must be constant, it is easily seen that along the surface dividing between resting and moving fluid, the so-called free boundary, the velocity has to be constant, that is, $\left(\nabla \phi^{\prime}\right)^{2}=$ const. Hence, under a $\delta n$-deformation which affects only the free boundary of the body $B^{\prime}$, we should have, by (3.16) and (3.17), $\delta M^{\prime}=\mu \delta V^{\prime}$. Thus, a free boundary leads to a stationary value for $M-\mu V$. One can now invert the reasoning and pose the extremum problem for $M-\mu V$ [18]. If one can show that a solu- 
tion exists and leads to a regular surface, one arrives at an existence proof for an interesting flow pattern. Such existence proof could be carried out indeed by interior variations in the case of plane flow [5] and of axially-symmetric flow [2]. The general case of a flow in three dimensions has not yet been solved satisfactorily.

The alternative approach to the free boundary problem for plane flows due to Leray [10] is based on fixed point theorems in function spaces and utilizes therefore also higher methods of functional analysis.

4. The second variation. The importance of the second variation for the theory of domain functionals is obvious. Consider a class of domains $D$ and a functional $\phi[D]$. Suppose now that any two domains $D_{0}$ and $D_{1}$ of the class can be connected by a continuously varying set of domains $D_{t}$ of the class with $0 \leqq t \leqq 1$. Then $\phi\left[D_{t}\right]$ becomes a function of the variable $t$. Suppose further that we can assert $d^{2} \phi_{t} / d t^{2}$ $>0$ if $D_{0} \neq D_{1}$. In this case, it is clear that there can exist only one domain in the class for which $\delta \phi=0$ for all admissible variations and which yields, consequently, a minimum of $\phi$. In many cases, it is more important to know that the functional equation implied by $\delta \phi \equiv 0$ has a unique solution than to know the exact value of the minimum. This is, for example, the case if the minimum problem has been set up artificially in order to obtain an existence proof as in the free boundary problem of the preceding section. In such cases, the knowledge of the second variation is most useful. Often it will even be sufficient to show that $d^{2} \phi_{t} / d t^{2}>0$ only in the case that $\delta \phi=0$ in order to make analogous deductions.

As indicated already in $\S 3$, it is of ten easy to find the higher order variations for functionals if we use the method of interior variations. Returning to the special case of the three-dimensional harmonic Green's function, we know already that $g(x, \xi ; \epsilon)$ is analytic in $\epsilon$ and that we can derive formally all coefficients in the power series development in $\epsilon$, obtaining finally all desired variations for the Green's function. We may express, for example, the second variation of $G(x, \xi)$ in terms of a triple integral over the domain $D$ involving the vector field $S_{i}(x)$ and the Green's function itself. We know, however, $a$ priori that the variation of $G$ must depend only on the shift of the boundary $C$ of $D$ and not on the interior values of the field; for the deformation of $C$ alone determines the varied domain $D^{*}$ with the Green's function $G^{*}$. Thus, it must be possible to express the second variation of the Green's function in the form of a surface integral over $C$, provided only that the boundary surface $C$ of $D$ is smooth 
enough. Similar arguments hold, of course, also in the case of variations of higher order.

We can give an elegant formula for the second variation of $G(x, \xi)$ in the case that the boundary surface $C$ is three times continuously differentiable. We consider a fixed vector field $S_{i}$ and denote by $G_{\epsilon}(x, \xi)$ the Green's function of the domain $D_{\epsilon}$ which arises from $D=D_{0}$ through the variation (3.1). We define $G(x, \xi)=G_{0}(x, \xi)$. Let

$$
N=\boldsymbol{S} \cdot \boldsymbol{n} ;
$$

$N$ is defined on $C$ and $n$ is the exterior normal on $C$ with respect to $D$. By Hadamard's formula (3.6), we have

$$
H(x, \xi)=\left.\frac{\partial}{\partial \epsilon} G_{\epsilon}(x, \xi)\right|_{\epsilon=0}=\iint_{C} \frac{\partial G(\eta, x)}{\partial n} \frac{\partial G(\eta, \xi)}{\partial n} N(\eta) d \sigma .
$$

Here, $H(x, \xi)$ is regular harmonic in $D$ and a linear functional of the vector field $\boldsymbol{S}$ on the boundary $C$. We can prove [3]:

$$
\begin{aligned}
\left.\frac{\partial^{2}}{\partial \epsilon^{2}} G_{\epsilon}(x, \xi)\right|_{\epsilon=0}= & -2 \iiint_{D}\left(\nabla_{\eta} H(\eta, x) \cdot \nabla_{\eta} H(\eta, \xi)\right) d \eta_{1} d \eta_{2} d \eta_{3} \\
& -\iint_{C} \frac{\partial G(\eta, x)}{\partial n} \frac{\partial G(\eta, \xi)}{\partial n} k(\eta) N^{2}(\eta) d \sigma,
\end{aligned}
$$

where $k(\eta)=1 / \rho_{1}+1 / \rho_{2}$ is the mean curvature of $C$ at the point $\eta$. This formula holds under the additional assumption that the vector field $S$ has on $C$ exact normal direction; otherwise some additional terms will come in depending on the tangential components of $\mathbf{S}$. Such effect of the tangential deformation was to be expected in the theory of the second variation; for, if we displace each boundary point in direction of the tangent plane, we do not affect the domain in the first order, but we must expect an influence of the second order variation.

The above formula for the second variation can be simplified considerably in important special cases. Consider, for example, a closed surface $T$ which encloses the boundary $C$ of $D$. Let $V(x)$ be harmonic in the shell between $C$ and $T$ and let $V=0$ on $C, V=1$ on $T$. In other words, $V(x)$ is the potential of the conductor made up of the surfaces $C$ and $T$. The level surfaces

$$
V(x)=t, \quad 0 \leqq t \leqq 1,
$$

are a continuously varying sequence of surfaces $C_{t}$ between $C$ and $T$. Let $G_{t}(x, \xi)$ be the Green's function of the domain $D_{t}$ interior to $C_{t}$. It is then easily shown that 


$$
\begin{aligned}
& \frac{\partial^{2}}{\partial t^{2}} G_{t}(x, \xi) \\
& \quad=-2 \iiint_{D_{t}}\left(\nabla_{\eta} \frac{\partial}{\partial t} G_{t}(\eta, x) \cdot \nabla_{\eta} \frac{\partial}{\partial t} G_{t}(\eta, \xi)\right) d \eta_{1} d \eta_{2} d \eta_{3} .
\end{aligned}
$$

We observe that

$$
G_{t}(x, \xi)=\frac{1}{4 \pi r(x, \xi)}-\Gamma_{t}(x, \xi), \quad r=\left(\sum_{i=1}^{3}\left(x_{i}-\xi_{i}\right)^{2}\right)^{1 / 2},
$$

where $\Gamma_{t}(x, \xi)$ is regular harmonic in the entire domain $D$. Since the singularity term is independent of the domain,

$$
\frac{\partial G_{t}}{\partial t}=-\frac{\partial \Gamma_{t}}{\partial t}, \quad \frac{\partial^{2} G_{t}}{\partial t^{2}}=-\frac{\partial^{2} \Gamma_{t}}{\partial t^{2}} .
$$

Hence, we find

$$
\begin{aligned}
\frac{\partial^{2}}{\partial t^{2}}\left[\sum_{i, k=1}^{N} \alpha_{i} \alpha_{k} \Gamma_{t}\left(x^{(i)}, x^{(k)}\right)\right] & \\
& =2 \iiint_{D t}\left(\nabla_{\eta} \sum_{i=1}^{N} \alpha_{i} \frac{\partial \Gamma_{t}\left(\eta, x^{(i)}\right)}{\partial t}\right)^{2} d \eta_{1} d \eta_{2} d \eta_{3} \geqq 0
\end{aligned}
$$

for any choice of the constants $\alpha_{i}$ and of the points $x^{(i)}$ in $D$. We arrive thus at a very general convexity theorem in potential theory and the applications indicated at the beginning of this section become possible.

Sometimes we can utilize the formula for the second variation more effectively than by just using the fact that it is positive. Take, for example, the functional $K[B]$, the electrostatic capacity of the body $B$; it is defined as follows: Let $\psi(x)$ be harmonic in the outside $D$ of $B$ and have the boundary value 1 on $C$. Then, $\psi(x)$ is called the conductor potential of $B$. It has at infinity the behavior

$$
\psi=\frac{K[B]}{r}+o(1 / r), \quad r^{2}=\sum_{i=1}^{3} x_{i}^{2}
$$

and this serves to define the electrostatic capacity $K[B]$.

Let now $C_{t}$ be a sequence of surfaces connecting continuously the surface $C$ of $B$ with another enclosing surface $T$ and being the level lines (4.4) of a harmonic function $V(x)$. The $C_{t}$ bound bodies $B_{t}$ with exterior $D_{t}$, capacity $K_{t}$, and conductor potential $\psi_{t}$. We can show from (4.5) that 


$$
\frac{\partial^{2}}{\partial t^{2}} K_{t}=\frac{1}{2 \pi} \iiint_{D_{t}}\left(\nabla \frac{\partial \psi_{t}}{\partial t}\right)^{2} d x_{1} d x_{2} d x_{3} .
$$

This shows that $K_{t}$ is convex from below as a function of $t$. But we can estimate the right-hand integral better if we observe that $h(x)=\partial \psi_{t} / \partial t$ is harmonic in $D_{t}$. Then, we have by Green's identity

$$
\iiint_{D t}\left(\nabla h \cdot \nabla \psi_{t}\right) d x_{1} d x_{2} d x_{3}=-\iint_{C_{t}} \frac{\partial h}{\partial n} d \sigma=4 \pi a
$$

if $\lim _{r \rightarrow \infty} h r=a$. Hence, by Schwarz's inequality

$$
(4 \pi a)^{2} \leqq \iiint_{D t}(\nabla h)^{2} d x_{1} d x_{2} d x_{3} \cdot \iiint_{D t}\left(\nabla \psi_{t}\right)^{2} d x_{1} d x_{2} d x_{3} .
$$

Finally, by applying (4.11) for the case $h=\psi_{t}$ and using (4.9), we find

$$
\iiint_{D_{t}}\left(\nabla \psi_{t}\right)^{2} d x_{1} d x_{2} d x_{3}=4 \pi K_{t}
$$

From (4.9) we deduce that $\lim _{r \rightarrow \infty} r\left(\partial \psi_{t} / \partial t\right)=\left(\partial K_{t} / \partial t\right)$ and hence (4.12), (4.13) yield

$$
\iiint_{D_{t}}\left(\nabla \frac{\partial \psi_{t}}{\partial t}\right)^{2} d x_{1} d x_{2} d x_{3} \geqq \frac{4 \pi}{K_{t}}\left(\frac{\partial K_{t}}{\partial t}\right)^{2}
$$

Hence, (4.10) leads to the differential inequality

$$
K_{t} \partial^{2} K_{t} / \partial t^{2} \geqq 2\left(\partial K_{t} / \partial t\right)
$$

which means that $K_{t}^{-1}$ is convex from above as a function of $t$.

This result is the best possible; for in the case that $T$ is a level surface $\psi(x)=$ const. of the conductor potential of $B$, it is easily seen that $K_{t}^{-1}$ is linear in $t$.

As another application, we consider two convex curves $C_{0}$ and $C_{1}$ in the plane which are described by their supporting functions $p_{0}(\phi)$ and $p_{1}(\phi)$. We can connect both curves by a continuous sequence of convex curves $C_{t}$ which are determined by the supporting functions,

$$
p_{t}(\phi)=(1-t) p_{0}(\phi)+t p_{1}(\phi) .
$$

In this case, the theory of the second variation yields the formula

$$
\begin{aligned}
& \frac{\partial^{2}}{\partial t^{2}} G_{t}(x, \xi) \\
& \leqq-2 \iint_{D t}\left(\nabla_{\eta} \frac{\partial}{\partial t} G_{t}(\eta, x) \cdot \nabla_{\eta} \frac{\partial}{\partial t} G_{t}(\eta, \xi)\right) d \eta_{1} d \eta_{2} d \eta_{3}
\end{aligned}
$$


where $G_{t}$ is the Green's function of the interior $D_{t}$ of $C_{t}$. This result leads again to numerous applications and convexity theorems in conformal mapping.

A main problem in applying the theory of the second variation is to find a convenient parametrization which permits us to connect any two domains of the class considered and which leads, on the other hand, to a simple second derivative of the functional in question with respect to that parameter.

5. Transplantation. Till now, we dealt mostly with functionals which arise from certain boundary value problems in partial differential equations. We consider now the important subclass of functionals which are defined as the minimum value of a given positivedefinite integral extended over the domain considered and depending on functions of a prescribed function class with respect to this domain. The fact that the functional is an actual minimum allows certain estimates by use of competing functions which lead to interesting inequalities and even to variational formulas.

We explain the method for the case of the electrostatic capacity $K[B]$ of a body $B$ with boundary surface $C$ and exterior $D$. We can characterize $K[B]$ by the following extremum properties $[13 ; 14]$ :

1. Dirichlet's principle: Consider all functions $U(x)$ which have continuous derivatives in $D$, are of order $O(1 / r)$ at infinity, and have on $C$ the boundary value one. Then

$$
K[B]=\min \frac{1}{4 \pi} \iiint_{D}(\nabla U)^{2} d x_{1} d x_{2} d x_{3}
$$

and the minimum is attained for the conductor potential $\psi(x)$ of $B$.

Dirichlet's principle leads to easy upper bounds for $K[B]$ since we may choose any permissible function $U(x)$ and find an inequality for $K[B]$.

2. Thomson's principle: Consider all vector fields $\boldsymbol{q}(x)$ in $D$ with continuously differentiable components, which are solenoidal, that is, satisfy $\nabla \boldsymbol{q}=0$, are normalized by

$$
\iint_{C} \mathbf{q} \cdot \mathbf{n} d \sigma=1
$$

and vanish at infinity such that $|q|=O\left(r^{-2}\right)$ there. Then

$$
\frac{1}{4 \pi K[B]}=\min \iiint_{D} \mathbf{q}^{2} d x_{1} d x_{2} d x_{3}
$$

and the minimum is attained by the vector field $q=(1 / 4 \pi K) \nabla \psi$. 
Thomson's principle leads to lower bounds for $K[B]$ and is very useful in combination with the preceding Dirichlet principle.

3. Gauss' principle: Consider all functions $\mu(P)$ defined on the boundary surface $C$ with the normalization

$$
\iint_{C} \mu d \sigma=1
$$

Then

$$
\frac{1}{K}=\min \iint_{C} \iint_{C} \mu(x) \mu(\xi) r(x, \xi)^{-1} d \sigma_{x} d \sigma_{\xi}
$$

and the minimum value is attained for $\mu(x)=-(1 / 4 \pi K) \partial \psi / \partial n$.

Gauss' principle stresses the fact that the capacity is ultimately a functional of the surface $C$ only. In physical interpretation, it expresses the capacity in terms of electric charges rather than in terms of electric fields.

We start now with a body $B=B(1)$ and consider the transformation of the $x$-space

$$
x_{1}^{\prime}=t x_{1}, \quad x_{2}^{\prime}=x_{2}, \quad x_{3}^{\prime}=x_{3} ;
$$

this is a stretching in the $x_{1}$-direction in the ratio $t: 1$. The body $B(1)$ is deformed continuously through a sequence of bodies $B(t)$ with exteriors $D(t)$, boundaries $C(t)$, conductor potentials $\psi_{t}(x)$, and capacities $K(t)$. Since $\psi_{t_{0}}(x)$ is defined in $D\left(t_{0}\right)$, clearly

$$
U(x)=\psi_{t_{0}}\left(\frac{t_{0}}{t} x_{1}, x_{2}, x_{3}\right)
$$

is well-defined in $D(t)$. It has the boundary value 1 on $C(t)$ and the correct continuity and asymptotic behavior at infinity in order to be admissible in the Dirichlet principle (5.1) with respect to $D(t)$. Thus, we obtain

$$
\begin{aligned}
K(t) \leqq \frac{1}{4 \pi} \iiint_{D(t)}\left[\left(\frac{t_{0}}{t}\right)^{2} \psi_{t_{0} ; 1}\left(\frac{t_{0}}{t} x_{1}, x_{2}, x_{3}\right)^{2}\right. & \\
& \left.+\psi_{t_{0} ; 2}^{2}+\psi_{t_{0} ; 3}^{2}\right] d x_{1} d x_{2} d x_{3}
\end{aligned}
$$

where $\psi_{t_{0 ;} ;}$ denotes the partial derivative of $\psi_{t_{0}}$ with respect to its $i$ th variable. We may refer back the integration to $D\left(t_{0}\right)$ by replacing $\left(t_{0} / t\right) x_{1}$ by $x_{1}$. This will change also the volume element in the integral and (5.8) obtains the form 


$$
t^{-1} K(t) \leqq \frac{1}{4 \pi t_{0}} \iiint_{D\left(t_{0}\right)}\left[\frac{t_{0}^{2}}{t^{2}} \psi_{t_{0} ; 1}^{2}+\psi_{t_{0} ; 2}^{2}+\psi_{t_{0} ; 3}^{2}\right] d x_{1} d x_{2} d x_{3}
$$

where the arguments of all $\psi_{t_{0} ; i}$ are now $x_{1}, x_{2}, x_{3}$. Thus, we may put this inequality into the form

$$
t^{-1} K(t) \leqq t^{-2} A+B
$$

where $A$ and $B$ depend on $t_{0}$ but not on $t$. Moreover

$$
\bar{t}_{0}^{-1} K\left(t_{0}\right)={\overline{t_{0}}}^{-2} A+B \text {. }
$$

Hence, if we plot $t^{-1} K(t)$ versus $\tau=t^{-2}$, we see that the straight line $a \tau+B$ touches this curve at the point $\tau_{0}=t_{0}^{-2}$ but lies elsewhere always above it. Hence, the curve $t^{-1} K(t)$ possesses at $\tau_{0}$ a supporting line from above. Since $t_{0}$ is arbitrary, we proved that $t^{-1} K(t)$ is convex from above as a function of $t^{-2}$. If we did not know already from the general theory that $K(t)$ is analytic in $t$, we could deduce at least from the convexity result that $K(t)$ has a derivative in $t$ almost everywhere. We can easily derive

$$
K^{\prime}(t)=\frac{1}{4 \pi t} \iiint_{D(t)}\left[\psi_{t 2}^{2}+\psi_{t 3}^{2}-\psi_{t 1}^{2}\right] d x_{1} d x_{2} d x_{3} ;
$$

and the differential inequality stating the above convexity

$$
t^{2} K^{\prime \prime}(t)+t K^{\prime}(t) \leqq K(t) .
$$

The basic idea in the above procedure is the transplantation of the correct extremum function from its domain $D\left(t_{0}\right)$ into another domain $D(t)$ where it serves as a comparison function in the extremum principle and leads thus easily to an inequality between the functionals of $D\left(t_{0}\right)$ and $D(t)$. This method of transplanting the extremum function has been used extensively in the following form. One uses one distinguished domain $D_{0}$, which is suspected to be the extremum domain, and its extremum function. Transplating this extremum function into all admissible domains $D$ of the class considered one shows that the functional $\phi[D]$ by its minimum property is less than the value achieved by the transplanted function and moreover that this latter value is less than $\phi\left[D_{0}\right]$. This shows that $\phi\left[D_{0}\right]>\phi[D]$ and establishes the extremum property of $D_{0}[13]$.

The difference between this well known and useful device and the above method is that in the latter we transplant the extremum function of each domain into each other domain; in this way, we obtain convexity statements and differential inequalities [14]. 
We can repeat the procedure in the case of the Thomson principle. Here, we have to deal with an extremum vector field instead of an extremum function. Let $\boldsymbol{q} \equiv\left(q_{1}, q_{2}, q_{3}\right)$ be the extremum vector field for the exterior $D\left(t_{0}\right)$ of the body $B\left(t_{0}\right)$. Then

$$
\begin{aligned}
q_{t} \equiv\left(q_{1}\left(\frac{t_{0}}{t} x_{1}, x_{2}, x_{3}\right), \frac{t_{0}}{t} q_{2}\left(\frac{t_{0}}{t} x_{1}, x_{2}, x_{3}\right)\right. & \\
& \left.\frac{t_{0}}{t} q_{3}\left(\frac{t_{0}}{t} x_{1}, x_{2}, x_{3}\right)\right)
\end{aligned}
$$

will be defined in $D(t)$ and represent a solenoidal vector field there. It is easily seen that the normalization (5.2) is transplanted also. Hence, we may use $\boldsymbol{q}_{t}$ in order to obtain a lower bound for $K(t)$. An easy calculation shows that $t K(t)^{-1}$ is convex from above as a function of $t^{2}$. This leads to another differential inequality which combines with (5.11) to estimate $K^{\prime \prime}(t)$ from above and from below. We obtain the inequality

$$
\left(a t^{2}+b\right)^{-1} \leqq t^{-1} K(t) \leqq A t^{-2}+B
$$

where equality holds on both sides for $t=t_{0}$. Thus, the curve $t^{-1} K(t)$ lies between two differentiable curves which touch for $t=t_{0}$. Clearly, $t^{-1} K(t)$ must be also differentiable there and since $t_{0}$ is quite arbitrary, we have proved that $K(t)$ is differentiable in $t$ everywhere. Thus, combining the Dirichlet with the Thomson principle we obtained a much stronger result than could have been obtained from each of them.

Finally, we may utilize the Gauss principle by transplanting the charge density $\mu d \sigma$ in the deformation. This leads to the result that $t K(t)^{-1}$ is convex from above as a function of $t$. This result is an improvement on the convexity statement derived from the Thomson principle. It is also easy to read off from the Gauss principle that $K(t)$ increases with $t$; in fact, under transplantation corresponding charges are pulled away from each other and the energy of the charge distribution is clearly decreased and the capacity $K$ is, consequently, increased. On the other hand, $K^{\prime}(t)$ is given by $(5.10)$ and it is by no means clear that this integral must be non-negative. But the combination of the two results leads to the inequality

$$
\iiint_{D} \psi_{x_{1}}^{2} d x_{1} d x_{2} d x_{3} \leqq \iiint_{D}\left[\psi_{x_{2}}^{2}+\psi_{x_{3}}^{2}\right] d x_{1} d x_{2} d x_{3}
$$

valid for every conductor potential and arbitrary choice of the coordinate system. Equality holds in (5.13) if the body $B$ is a plate in a 
plane $x_{1}=$ const. In fact, such a plate is not affected by a stretching in the $x_{1}$-direction and hence $K^{\prime}(t) \equiv 0$.

It seems that the transformation by stretching is a very special kind of deformation and that the results obtained in this way are rather restricted. Before discussing other and more general deformations which admit a similar treatment, we want to show a slight generalization of the stretching deformation which leads to rather important applications. We consider the transformation

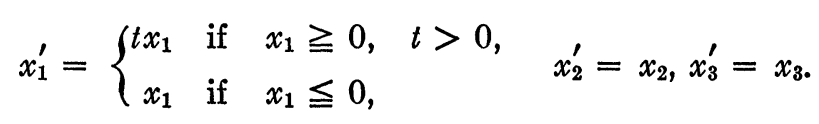

This is a continuous transformation of the $x$-space, but it is no longer continuously differentiable. We call it a partial stretching in the $x_{1^{-}}$ direction.

If we want to apply the transplantation method to the capacity $K[B]$ in the case of a partial stretching, we have to observe, at first, that the transplanted functions and vector fields have discontinuities or discontinuous derivatives along the plane $x_{1}=0$. However, it can be seen that Dirichlet's and Thomson's principles still hold for extended classes of functions or vector fields, which include the transplanted fields obtained. Hence, it can be shown, as before, that $t^{-1} K(t)$ is convex from above in $t^{-2}$ while $t K(t)^{-1}$ is convex from above in $t^{2}$, even in the case of a partial stretching. The derivative of $K(t)$ with respect to $t$ can be shown to be

$$
K^{\prime}(t)=\frac{1}{4 \pi t} \iiint_{D(t)}\left[\psi_{t 2}^{2}+\psi_{t 3}^{2}-\psi_{t 1}^{2}\right] d x_{1} d x_{2} d x_{3}
$$

where $D(t)+$ is the intersect of $D(t)$ with the half-space $x_{1} \geqq 0$.

Since we may put the axes of reference arbitrarily with respect to the body considered, we obtain a great variety of deformations of the original body under partial stretching. In particular, we may put the plane $x_{1}=0$ in such a way that it cuts off only a very small part of the body $B$ so that the partial stretching becomes a variation of a surface element of $B$. Using our above results, and in particular (5.15), we obtain a new approach to variational formulas of the Hadamard type.

Other interesting applications of the transplantation method arise in eigenvalue problems. In the membrane equation

$$
\nabla^{2} u+\lambda u=0,
$$$$
u=0 \text { on } C
$$

we can characterize the eigenvalues by well known extremum prin- 
ciples. We restrict ourselves here to the lowest eigenvalue $\lambda_{1}(D)$ of a domain $D$ in the $(x, y)$-plane. Let $u(x, y)$ be any continuously differentiable function in $D$ which vanishes on the boundary $C$; then we have

$$
\lambda_{1}=\min \frac{\iint_{D}(\nabla u)^{2} d x d y}{\iint_{D} u^{2} d x d y} .
$$

The minimum is attained by the eigenfunction of (5.16) to the eigenvalue $\lambda_{1}$.

It is easy to find upper bounds for the eigenvalue $\lambda_{1}$ because of its minimum definition; it is, in general, not quite as easy to derive lower bounds. Here, convexity statements for the eigenvalue in dependence on some parameter $t$ for a sequence of domains $D(t)$ may be of great value.

We can easily show that under a stretching

$$
x^{\prime}=t x, \quad y^{\prime}=y
$$

we obtain a sequence of domains $D(t)$ with the $n$th eigenvalue $\lambda_{n}(t)$ such that $\sum_{\nu=1}^{N} \lambda_{\nu}(t)$ is a monotonic function of $t^{-2}$, convex from above; this holds for every choice of the integer $N$. Observe that the sum considered is not necessarily continuously differentiable in $t$; it may have discontinuous derivatives for the values of the parameter $t$ for which $\lambda_{N}(t)$ is a degenerate eigenvalue. The same result holds for the eigenvalues of the more general differential system

$$
\nabla^{2} u+\lambda u=0, \quad \frac{\partial u}{\partial n}+k u=0 \text { on } C .
$$

These convexity results have been applied to estimate the eigenvalues for isosceles triangles from the known eigenvalues for the equilateral triangle and their asymptotic behavior for $t \rightarrow 0$ and $t \rightarrow \infty$ [12].

Consider next the sequence of domains $D(t)$ which are obtained from a given domain $D=D(0)$ by the conformal mappings

$$
z^{*}=z+t f(z)
$$

where $f(z)$ is analytic in $D(0)$. It is not necessary that all domains $D(t)$ lie schlicht over the complex plane; we may define their eigenvalues $\lambda_{n}(t)$ of the membrane problem even if they lie over a Riemann surface.

If $u(x, y)$ is a correct first eigenfunction of the membrane problem 
for the domain $D(0)$, we may consider $u(x, y)$ referred by $z\left(z^{*}\right)$ to $D(t)$ as a comparison function for the extremum problem. We may assume that $u$ was normalized in $D(0)$ by the condition

$$
\iint_{D(0)} u^{2} d x d y=1
$$

Observe that the Dirichlet integral in the numerator of (5.17) is invariant under conformal transformation. Thus, (5.17) applied to $D(t)$ leads to

$$
\begin{aligned}
\lambda_{1}(t)^{-1} & \geqq \lambda_{1}(0)^{-1} \iint_{D(0)} u^{2}(x, y)\left|1+t f^{\prime}(z)\right|^{2} d x d y \\
& \geqq \lambda_{1}(0)^{-1}+\lambda_{1}(0)^{-1} t \cdot 2 \operatorname{Re}\left\{\iint_{D(0)} u^{2} f^{\prime}(z) d x d y\right\} .
\end{aligned}
$$

This shows that $\lambda_{1}(t)^{-1}$ is convex from below as a function of $t$, since we can deduce in the same way the existence of a supporting line for every value of $t$.

Using the extremum definition for the higher eigenvalues and the same transplantation argument, we can prove that $\sum_{v=1}^{N} \lambda_{\nu}^{-1}(t)$ is convex from below as a function of $t$, for every choice of the integer $N$. This result leads to interesting inequalities between the eigenvalues of the membrane problem and conformal moduli of the domain $D[14]$.

An extension of the preceding convexity result to the more general system (5.19) seems not easy. The boundary conditions are only conformally invariant if $k=0$ and, in this case, we have $\lambda_{1}=0$. Hence, no corresponding result is known in the more general case (5.19).

We do not want to multiply examples, but mention that the transplantation method proved also useful in the theory of torsional rigidity, virtual mass, conformal radius, etc. [14].

It may be of interest to quote a result from the theory of differential equations of higher than the second order. Consider the differential equation

$$
\nabla^{4} u=\lambda u, \quad u=\frac{\partial u}{\partial n}=0 \text { on } C,
$$

for a plane domain $D$ with boundary $C$. Then, it can be shown that under a stretching (5.18) of $D$, the functional $\lambda(D)$ is convex from above as a function of $t^{-4}$.

6. General method. In the case that $\phi[D]$ is a functional defined 
as the minimum value of a positive-definite Dirichlet integral $Q[u]$ which is quadratic in its argument function $u$, we can develop a very simple variational theory. We assume that the minimum in $Q[u]$ is understood with respect to a well-defined function class $\mathfrak{F}$ characterized by certain continuity and boundary conditions with respect to $D$. Suppose that a transformation

$$
x_{i}^{*}=x_{i}+\epsilon S_{i}(x)
$$

is considered which carries $D=D(0)$ into a sequence of domains $D(\epsilon)$; we assume that the functions of the class $\mathfrak{F}$ are transformed by transplantation into $D(\epsilon)$ into functions of the corresponding function class $\mathfrak{F}_{\epsilon}$ of the new domain and vice versa. Thus, each admissible function $u^{*}\left(x^{*}\right)$ in $D(\epsilon)$ becomes admissible in $D(0)$ after transplantation.

The Dirichlet integrals $Q_{\epsilon}\left[u^{*}\right]$ for the various domains $D(\epsilon)$ become after transplantation into $D(0)$ well-defined Dirichlet integrals $Q[u ; \epsilon]$ and are to be considered over the function class $\mathfrak{F}$; clearly $Q[u ; 0]=Q[u]$.

Let $u(x ; \epsilon)$ be the extremum function which yields the minimum

$$
\phi(\epsilon)=Q[u(x ; \epsilon) ; \epsilon] \leqq Q\left[u_{0} ; \epsilon\right],
$$

$u_{0} \in \mathfrak{F}$,

within the class $\mathfrak{F}$. Obviously

$$
\phi(\epsilon)=\phi[D(\epsilon)]
$$

and the study of $\phi(\epsilon)$ in its dependence on the parameter $\epsilon$ leads to the complete variational theory of the functional $\phi[D]$.

Observe now that

$$
\begin{aligned}
\phi(\epsilon)-\phi(0)= & Q[u(x ; \epsilon) ; \epsilon]-Q[u(x ; \epsilon) ; 0] \\
& +Q[u(x ; \epsilon) ; 0]-Q[u(x ; 0) ; 0] \\
= & Q[u(x ; \epsilon) ; \epsilon]-Q[u(x ; 0) ; \epsilon] \\
& +Q[u(x ; 0) ; \epsilon]-Q[u(x ; 0) ; 0] .
\end{aligned}
$$

Since $u(x ; \epsilon)$ and $u(x ; 0)$ both belong to the class $\mathfrak{F}$, we have by the minimum properties $(6.2)$

$$
Q[u(x ; \epsilon) ; \epsilon] \leqq Q[u(x ; 0) ; \epsilon] ; \quad Q[u(x ; 0) ; 0] \leqq Q[u(x ; \epsilon) ; 0] .
$$

Thus, (6.4) leads to the inequalities

$$
\begin{aligned}
& Q[u(x ; \epsilon) ; \epsilon]-Q[u(x ; \epsilon) ; 0] \\
& \quad \leqq \phi(\epsilon)-\phi(0) \leqq Q[u(x ; 0) ; \epsilon]-Q[u(x ; 0) ; 0] .
\end{aligned}
$$


We have, until now, not made any assumption regarding the character of the quadratic Dirichlet integral $Q[u]$. We suppose that $Q[u ; \epsilon]$ has for fixed $u(x)$ a continuous derivative $Q_{1}[u ; \epsilon]$ with respect to $\epsilon$ which is again a quadratic functional of $u$. We have then by the mean value theorem

$$
Q\left[u ; \epsilon_{2}\right]-Q\left[u ; \epsilon_{1}\right]=\left(\epsilon_{2}-\epsilon_{1}\right) Q_{1}[u ; \eta]
$$

where $\eta$ is an intermediate value between $\epsilon_{1}$ and $\epsilon_{2}$. We restrict ourselves to the interval $|\epsilon| \leqq 1$ and assume further that $Q_{1}[u ; \epsilon]$ is there uniformly continuous as a functional of $u$ in the $Q[u]$-metric and that there exist positive constants $a, b, \alpha, \beta$ such that

$$
a Q[u] \leqq Q[u ; \epsilon] \leqq b Q[u] ; \quad \alpha Q[u] \leqq\left|Q_{1}[u ; \epsilon]\right| \leqq \beta Q[u] .
$$

In many important cases, it is possible to verify that all these assumptions are fulfilled.

We derive now from (6.6) and (6.7) the inequality

$$
Q_{1}\left[u(x ; \epsilon) ; \eta_{1}\right] \epsilon \leqq \phi(\epsilon)-\phi(0) \leqq Q_{1}\left[u(x ; 0) ; \eta_{2}\right] \epsilon
$$

where $\eta_{1}$ and $\eta_{2}$ lie between 0 and $\epsilon$. From our assumptions (6.8) follows clearly that

$$
\phi(\epsilon)-\phi(0)=O(\epsilon),
$$

that is, in particular, the continuity of $\phi(\epsilon)$ at $\epsilon=0$.

Next, we observe that

$$
\begin{aligned}
& Q[u(x ; \epsilon)]-Q[u(x ; 0)] \\
& \quad=\phi(\epsilon)-\phi(0)-(Q[u(x ; \epsilon) ; \epsilon]-Q[u(x ; \epsilon) ; 0])
\end{aligned}
$$

which leads by (6.7), (6.8), and (6.10) to

$$
Q[u(x ; \epsilon)]-Q[u(x ; 0)]=O(\epsilon) .
$$

But from the quadratic character of $Q[u]$ and the minimum property of $u(x ; 0)$ with respect to this Dirichlet integral, we have

$$
Q[u(x ; \epsilon)]-Q[u(x ; 0)]=Q[u(x ; \epsilon)-u(x ; 0)]=O(\epsilon) .
$$

Thus, $u(x ; \epsilon)$ converges to $u(x ; 0)$ in the $Q$-metric and, consequently, in view of the assumed continuity properties of $Q_{1}[u ; \epsilon]$, we find from (6.9)

$$
\lim _{\epsilon \rightarrow 0} \frac{\phi(\epsilon)-\phi(0)}{\epsilon}=Q_{1}[u(x ; 0) ; 0] .
$$

This proves the differentiability of the functional and yields an explicit variational formula. 
It is interesting to observe the role which the actual minimum character of $\phi[D]$ plays in our reasoning. Terms like

$$
\frac{1}{\epsilon}(Q[u(x ; \epsilon) ; 0]-Q[u(x ; 0) ; 0]),
$$

which occur in (6.4) and are easily estimated from the minimum property, require a detailed investigation of the dependence of $u(x ; \epsilon)$ on $\epsilon$ if the minimum property does not hold.

In important cases the value $u(x ; \epsilon)$ itself can be characterized by a minimum problem of the above type. In this case, the above method leads to the derivative of $u(x ; \epsilon)$ with respect to $\epsilon$ which, in turn, leads to the second derivative of $\phi[\epsilon]$. In the case of the harmonic Green's function, for example, such reasoning leads to the derivatives of all orders of $G_{\epsilon}(x, \xi)$ with respect to the parameter.

\section{REFERENCES}

1. M. Biernacki, Sur la représentation conforme des domaines linéairement accessibles, Prace Math. Fiz. vol. 44 (1936) pp. 293-314.

2. P. R. Garabedian, H. Lewy and M. Schiffer, Axially symmetric cavitational flow, Ann. of Math. vol. 56 (1952) pp. 560-602.

3. P. R. Garabedian and M. Schiffer, Convexity of domain functionals, Journal d'Analyse Mathématique vol. 2 (1953) pp. 281-368.

4. - - Variational problems in the theory of elliptic partial differential equations, Journal of Rational Mechanics and Analysis vol. 2 (1953) pp. 137-171.

5. P. R. Garabedian and D. C. Spencer, Extremal methods in cavitational flow, Journal of Rational Mechanics and Analysis vol. 1 (1952) pp. 359-409.

6. G. Golusin, Method of variations in the theory of conformal mapping, Mat. Sbornik N.S. vol. 19 (1946) pp. 203-236; vol. 21 (1947) pp. 83-117, 119-132.

7. J. Hadamard, Mémoire sur le problème d'analyse relatif a l'équilibre des plaques élastiques encastrées, Mémoires des Savants Etrangers vol. 33 (1908).

8. D. Hilbert, Grundzïge einer allgemeinen Theorie der linearen Integralgleichungen, Leipzig, 1912.

9. G. Julia, Sur une équation aux dérivées fonctionelles liées à la représentation conforme, Ann. École Norm. (3) vol. 39 (1922) pp. 1-28.

10. J. Leray, Les problèmes de représentation conforme d'Helmholtz; théorie des sillages et des proues, Comment. Math. Helv. vol. 8 (1935) pp. 149-180, 250-263.

11. K. Löwner, Untersuchungen über schlichte konforme Abbildunges des Einheitskreises I, Math. Ann. vol. 89 (1923) pp. 103-121.

12. G. C. Nooney, On the vibrations of triangular membranes, Technical Report No. 35, Office of Naval Research Contract N6ori-106 Task Order V, Stanford University.

13. G. Pólya and G. Szegö, Isoperimetric inequalities of mathematical physics, Princeton, 1951.

14. G. Pólya and M. Schiffer, Convexity of functionals by transplantation, Journal d'Analyse Mathématique, to appear.

15. H. Poincaré, Figures d'équilibre d'une masse fluide, Paris, 1902. 
16. R. de Possel, Zum Parallelschlitztheorem unendlich-vielfach zusammenhängender Gebiete, Göttinger Nachr. (1931) pp. 199-202.

17. J. W. Rayleigh, The theory of sound, 2d ed., London, 1894.

18. D. Riabouchinsky, Sur un probleme de variation, C. R. Acad. Sci. Paris vol. 185 (1927) pp. 840-841.

19. A. C. Schaeffer and D. C. Spencer, Coefficient regions for schlicht functions, Amer. Math. Soc. Colloquium Publications, vol. 35, New York, 1950.

20. M. Schiffer, Variational methods in the theory of conformal mapping, Proceedings of the International Congress of Mathematicians 1950, vol. II, pp. 233-240.

21. M. Schiffer and D. C. Spencer, Functionals of finite Riemann surfaces, Princeton, 1954.

22. M. Schiffer and G. Szegö, Virtual mass and polarization, Trans. Amer. Math. Soc. vol. 67 (1949) pp. 130-205.

23. O. Teichmüller, Ungleichungen zwischen den Koeffizienten schlichter Funktionen, Preuss. Akad. Wiss. Sitzungsber. Phys.-math. Klasse (1938) pp. 363-375.

StaNFord UnIVERSity 